\section{Cureus}

\title{
Fluorescence and Glioma Surgery
}

\author{
Antonio Salas $^{1}$, Yashar Kalani ${ }^{1}$ \\ 1. Division of Neurological Surgery, Barrow Neurological Institute
}

$\square$ Corresponding author: Antonio Salas, adsalas@scu.edu

Disclosures can be found in Additional Information at the end of the article

\section{Abstract}

The introduction of fluorescence-based techniques has revolutionized the microsurgical resection of brain tumors, especially gliomas. In this review, we summarize the results of application of 5-ALA to glioma surgery, highlighting results and opportunities for innovation.

Categories: Neurosurgery

Keywords: fluorescence, glioma surgery, 5-ala

\section{Introduction And Background}

Malignant glioma, the most common type of brain cancer, is a highly aggressive glial cell cancer that is subdivided into anaplastic glioma and glioblastoma, which is the more common of the two types [1]. Treatment for malignant glioma consists of resection surgery followed by adjuvant radiation and chemotherapy-usually in the form of temozolomide [1]. In order to assure the best possible outcome for patients, neurosurgeons strive to completely remove tumors while minimizing removal of healthy tissue; however, this process is complicated by the invasive nature of gliomas and the presence of vital brain tissue. Patients whose tumors are incompletely resected have poor prognoses compared to those whose tumors are completely removed. Therefore, delineation of tumor margins is imperative for the survival of patients. In standard surgery performed under white light, only $13-20 \%$ of glioma resections successfully remove the entire tumor [2]. This rate illustrates that neurosurgeons frequently have difficulty discerning between healthy and malignant tissue during surgery. In order to rectify this predicament, numerous tactics, especially those utilizing fluorescent markers, are being introduced to aid neurosurgeons in identifying tumor margins more efficiently intraoperatively. Before glioma resection surgery, preoperative images are taken by CT and MRI to guide the surgery; however, these images are obscured by brain shift-a phenomena that shifts regions of the brain slightly due to intraoperative events, such as tissue removal, brain swelling, and cerebrospinal fluid drainage. Due to the dynamic nature of brain tissue during surgery, various methods have been used to either recalibrate images of the brain during procedures or emphasize the tumor tissue intraoperatively. One way to counteract brain shift is through intraoperative imaging, especially by utilizing MRI and ultrasound images. Unfortunately, MRI is expensive and time consuming relative to the surgery [2-3]. Utilizing ultrasound imaging during surgery is cheaper, but one must be exceptionally skilled in ultrasound technique to obtain robust images during surgery, and the instruments can interfere greatly with surgery [3]. In order to aid neurosurgeons to delineate tumor margins in real-time surgery, fluorescenceguided surgery, especially by 5-aminolevulinic acid (5-ALA)-induced fluorescence, offers a robust solution to maximizing the efficacy of tumor resection surgery.

\section{Review}

Neurosurgery guided by 5-ALA-induced fluorescence enables surgeons to meticulously discern 
between malignant and healthy tissue intraoperatively, which increases the likelihood that they can completely remove tumors while maximizing the amount of healthy tissue retained. 5-ALA is a metabolite involved in the heme synthesis pathway that is converted into protoporphyrin IX (PpIX), a highly fluorescent intermediate. When exogenous 5-ALA is administered, tumor cells produce and accumulate PpIX at concentrations that allow them to fluoresce, while most healthy tissue does not build up PpIX to fluorescing levels [4]. The rate-limiting step for heme synthesis is the production of 5-ALA, which is constructed from an ALA synthase-catalyzed reaction between glycine and succinyl-CoA. ALA synthase is subject to feedback inhibition from 5-ALA; therefore, by exogenously administering 5-ALA, one circumvents this ratelimiting step-ultimately allowing for an overactivation of the heme synthesis pathway [4]. It is unclear what mechanism dictates the highly specific accumulation of PpIX in tumor tissue. Some speculate that because tumor cells down-regulate the activity of the ferrochelatase enzyme, which converts PpIX into the non-fluorescent heme product, malignant tissue is able to accumulate PpIX when 5-ALA is introduced exogenously, while healthy cells, by efficiently converting excess PpIX into heme, do not $[2,5]$. Under normal circumstances, 5-ALA, because it is not significantly lipid soluble, cannot cross the blood-brain barrier [4]. However, when the blood-brain barrier is compromised by tumor angiogenesis, 5-ALA is more permeable to the blood-brain barrier, thus contributing to 5-ALA's ability to target tumor tissue [2, 4]. In a study by Stummer, et al., the authors found that 5-ALA-induced fluorescence is highly correlated to the magnitude of cell proliferation and cellular density [4]. Therefore, because uncontrolled cell growth, which creates high cell density, is a major hallmark of cancer, this attribute could reflect why PpIX accumulates more in malignant tissue after 5-ALA administration. However, regardless of the mechanism involved, PpIX fluorescence from 5-ALA has the capacity to act as a guiding tool in glioma resection surgery due to its accurate ability to emphasize tumor tissue.

Studies on the efficacy of 5-ALA fluorescence-guided surgery show that the procedure significantly increases the likelihood that neurosurgeons will be able to remove as much tumor as possible. In order to evaluate 5-ALA as a viable tool to improve malignant glioma resections, a Phase III randomized clinical trial was conducted in Germany at 15 neurosurgical centers on 322 patients. In this study, patients underwent 5-ALA-induced fluorescence-guided surgery followed by adjuvant radiotherapy or the control protocol, which was radiotherapy after white light neurosurgery [2]. The study concluded that 5-ALA administration significantly increased neurosurgeons' ability to completely remove tumors (total resection in $65 \%$ of patients in the 5-ALA arm compared to 36\% of patients in the white light arm) and improved progression-free survival after six months (progression-free survival after six months was $41 \%$ in the 5-ALA arm compared to $21.1 \%$ in the white light arm). These results clearly illustrate that utilizing 5-ALA for fluorescence-guided surgery is a robust method of neurosurgical resection surgery for malignant glioma [2]. Ultimately, these positive results have led the European Union to implement 5-ALA administration in regimens for malignant glioma resection. Unlike Europe and Asia, 5-ALA has yet to be approved to aid malignant glioma neurosurgeries in the U.S. Studies have indicated that 5-ALA's ability to induce fluorescence in tumor tissue works exceedingly well in aggressive, high-grade gliomas, but 5-ALA does not identify low-grade glioma tumors adequately. In a study conducted on 59 patients with diffusely infiltrating gliomas who underwent 5-ALA fluorescence-guided surgery, 91\% of the patients with lowgrade gliomas (WHO Grade II) did not have fluorescence induced in their tumors [6]. On the other hand, in another study on patients with high-grade gliomas (WHO Grade III \& IV) that underwent 5-ALA-guided resection surgery, $100 \%$ of patients had at least $90 \%$ of the tumor removed and $93 \%$ of patients studied had $98-100 \%$ of their tumor removed [7]. It is evident that 5-ALA can be an extremely useful tool in resection surgeries involving high-grade gliomas, but due to its poor ability to induce fluorescence in Grade II gliomas, 5-ALA is not a viable option for guiding low-grade glioma resections. Overall, 5-ALA-induced fluorescence offers a safe, effective method of differentiating between malignant and healthy tissue during glioma resection surgery, especially for aggressive high-grade gliomas. It is apparent that 5-ALA fluorescence-guided surgery has demonstrated great success for resection surgery of malignant 
glioma, especially for more aggressive, high-grade gliomas.

Besides its obvious ability to delineate tumor margins during glioma resection surgery, 5-ALA's ability to induce fluorescence in tumors is capable of contributing to cancer therapy in numerous ways. Research on the benefits of using 5-ALA-induced accumulation of PpIX in tumors for photodynamic therapy and photodiagnosis have shown promising results in both fields [8-9]. Besides photodynamic therapy and photodiagnosis, 5-ALA-induced fluorescence can improve the accuracy of biopsies. Before an oncologist can decide the most appropriate method to treat malignant glioma patients, especially concerning immediate postoperative therapy, they must evaluate the histology of the tumor tissue. For low-grade glioma treatment, complete resection surgery is the sole therapy until tumorigenesis progresses [6]. On the other hand, for high-grade gliomas, postoperative radiochemotherapy must immediately follow resection surgery [6]. Therefore, it is imperative that robust biopsies are taken in order to appropriately treat patients. Research suggests that utilizing 5-ALA-induced fluorescence to guide biopsies yields more accurate biopsies with samples taken during surgery and with frameless MRI stereotactic biopsies [6, 10]. Not only can PpIX fluorescence be utilized for resections and biopsies, 5-ALA has the capacity to guide resection surgeries of recurrent malignant gliomas. A Phase II, multicenter, prospective, single-arm, uncontrolled study conducted on 36 patients to evaluate the viability of 5-ALA for fluorescence-guided surgery on recurrent glioma showed promising results. After resection surgery, biopsies of non-fluorescing and fluorescing areas were taken to evaluate the accuracy of 5-ALA to identify healthy and malignant tissue. The positive predictive value (PPV), which indicates the frequency of correctly identified tumors, of the samples displayed a 96.6\% PPV [2]. Thus, these results indicate that 5-ALA fluorescence-guided surgery accurately resects recurrent glioma tumors. Due to this procedure's Phase II status, however, it must still undergo significant research before it can be implemented into contemporary surgical regimens. On the other hand, 5-ALA fluorescence-guided surgery for recurrent malignant glioma has been approved in Europe, the Middle East, and Africa (EMEA). It is apparent that 5-ALA is beneficial for recurrent resection surgery of malignant glioma tumors. Overall, besides its ability to improve the efficacy of gliomal resection surgery, 5-ALA, due to its ability to induce fluorescence in tumor tissue, is capable of improving malignant gliomal therapy in a myriad of ways.

The usual procedure of utilizing 5-ALA for resection surgery consists of administering $20 \mathrm{mg} / \mathrm{kg}$ body weight of 5-ALA orally to patients two to four hours prior to the procedure [4-5, 10]. Preclinical data suggests that 5-ALA-induced fluorescence can last up to 12 hours and is optimal two to eight hours after it is administered [10-11]. In order to observe the fluorescence, the region of interest must be illuminated with a blue xenon light $(440 \mathrm{~nm})$ to the excite PpIX molecules, allowing them to fluoresce in a red or pink color. Most of the adverse effects-such as progression of hemiparesis, pulmonary thromboembolism, and thrombocytopenia-associated with 5-ALA fluorescence-guided surgery are observed in all glioma resection surgeries. On rare occasions, patients experience nausea, vomiting, and hypotension after 5-ALA administration. Patients have a heightened sensitivity to sunlight exposure for 48 hours after administration of 5-ALA due to the surplus of PpIX in their skin [5]. In addition, prior adjuvant therapy does not influence the ability for 5-ALA to induce fluorescence in tumors. Overall, 5-ALA fluorescenceguided surgery is a safe and easy method for significantly improving neurosurgeons' ability to discern between tumor and healthy tissue intraoperatively.

It is apparent that 5-ALA administration is a successful method of marking tumor tissue during surgery; unfortunately, the procedure has its shortcomings. For an unknown reason, a small number of tumors do not fluoresce when 5-ALA is administered [2]. Due to heterogeneous tumor cell populations, some portions of malignant tissue fluoresce more than others, which makes discerning between cancerous and healthy tissue more difficult [2]. Also, 5-ALA-induced fluorescence works most efficiently on more aggressive high-grade gliomas. Because xenon lights are necessary to excite PpIX, issues pertaining to lighting setup, such as shading and 
distance from light to tumor, and the age of the light could obscure neurosurgeons' ability to evaluate tissue. Unfortunately, PpIX fluorescence is also prone to photobleaching, which degrades PpIX due to prolonged exposure to excitation light. In addition, xenon lights are only capable of penetrating to a depth of $1 \mathrm{~mm}$; therefore, small amounts of blood could easily block a tumor tissue getting excited [2]. Although 5-ALA-induced fluorescence-guided surgery is a robust system for accurately resecting tumors, it does possess pitfalls.

Besides 5-ALA-induced fluorescence, which is an exogenously induced fluorescence procedure, other methods (endogenous autofluorescence and exogenous contrast agents) are capable of using fluorescence to emphasize tumor tissue in real-time surgery [8]. Endogenous autofluorescence, a technique that utilizes UV and blue light excitation to emphasize tumor tissue, can also be exploited for tumor resection surgery. After exciting tumor tissue, the emission spectra are visualized in the blue-green spectra. The fluorescent signals are derived from collagen, NADH, and FADH for unknown reasons [8]. By utilizing endogenous fluorescence signals, medical shortcomings, such as allergic reactions, are avoided. Because it is not necessary to introduce an exogenous fluorescent agent, procedures using endogenous fluorescence require little effort. Due to the lack of a contrasting agent, the ability to delineate tumor margins could be obscured by the lack of tumor background contrast. Unfortunately, the synergy of blood absorption, light scatter, and tissue layers obscure the neurosurgeon's ability to interpret the spectra intraoperatively [8]. Also, this method requires that the data, which takes up to 30 seconds to acquire, be analyzed postoperatively [8]. The pilot studies have shown promising results for autofluorescence $[8,12]$. In a study on 24 brain tumor patients, the researchers used autofluorescence combined with diffuse-reflectance spectroscopy to differentiate between tumor and healthy tissue, and they found that this method yielded 100\% sensitivity and $76 \%$ specificity [12]. These results suggest that autofluorescence can be a potential tool in fluorescence-guided surgery due to its accurate ability to identify tumor tissue; however, due to the relatively low specificity observed, this method could potentially influence neurosurgeons to resect ample healthy tissue. The efficacy of endogenous fluorescence-guided surgery must be studied further in order to implement the technique into neurosurgical regimen because it is still in the pilot study phase of research.

Besides endogenous autofluorescence and exogenously induced fluorescent techniques for fluorescence-guided surgery, neurosurgeons can utilize exogenous contrast agents to identify tumor tissue through fluorescence in real-time surgery. Fluorescein sodium, a small organic molecule that fluoresces a yellow-green color in white light, is an exogenous contrast agent that was first introduced in tumor resection surgery in 1948. It has been used extensively for the past couple of decades for brain tumor identification during open biopsies and retinal angiography $[8,13]$. Similarly to 5-ALA, fluorescein sodium can target malignant tissue because it is permeable to regions of the brain where the blood-brain barrier is distorted from tumor angiogenesis. Because fluorescein sodium administration allows brain tumors to fluoresce in white light, resection surgeries can proceed in dim light devoid of extra surgical equipment. Unfortunately, fluorescein sodium administration can have adverse side effects, especially if it is rapidly administered or given at high concentrations [8]. Additionally, neurosurgeons can utilize intraoperative confocal endomicroscopes with fluorescein sodium, 5-ALA, and indocyanine green to enhance the fluorescent intensity, which improves one's ability to delineate tumor margins. In a Phase II prospective study on the efficacy of fluorescein sodium in conjunction with a surgical microscope fitted with filters to emphasize fluorescing tissue for fluorescence-guided surgery on 20 glioma patients, $80 \%$ of patients had their tumors completely removed, and six month progression-free survival was 71.4\% [13]. Thirty-six biopsies of fluorescing and non-fluorescing samples (18 samples for each group) taken at tumor margins were evaluated by immunohistochemical analysis and reflected robust results (94\% sensitivity and $89.5 \%$ specificity). In order to use indocyanine green as a fluorescing agent, neurosurgeons must use endoscopes, microscopes, or other imaging techniques that are tailored to view near-infrared fluorescence. Also, PpIX fluorescence from 5-ALA can be 
detected in individual cells using a confocal endomicroscope, which allow tumor margins to be identified more easily; however, photobleaching occurs quickly (36\% with 25 minutes of excitement) [3]. Overall, using fluorescein sodium, especially in conjunction with endomicroscopes equipped with the appropriate filters, to guide glioma resection surgeries could offer a robust strategy to maximize the amount of tumor tissue removed; however, Phase III clinical trials must be conducted to appropriately evaluate the viability of using this technique.

Besides fluorescein sodium, exploiting indocyanine green, a small water-soluble organic compound with a near-infrared spectral peak, could offer a promising strategy of providing fluorescence for fluorescence-guided surgery intraoperatively. Near-infrared fluorescence confocal microscopy techniques, such as the exploitation of indocyanine green as a contrast medium, have greater imaging depth than visible-wavelength fluorescence confocal microscopy methods, such as 5-ALA and fluorescein sodium methods [14]. Because of this, indocyanine green potentially can be very useful for accurately identifying tumor margins. In order to exploit indocyanine green's ability to emphasize tumor margins and tumor tissue, a neurosurgeon must utilize a confocal endomicroscope or surgical microscope that is tailored to view near infrared images. Because of its greater imaging depth, near infrared systems can be superior to visible wavelength fluorescent methods for confocal microscopic identification of tumor margins. On one hand, sodium fluorescein is impermeable to cells, provides a fluorescent background immediately, and its fluorescence diminishes as it is exposed to lasers and lights [3]. On the other hand, indocyanine green provides a greater ability to identify malignant margins than fluorescein, is less toxic than fluorescein, is permeable to cells, and continues to fluoresce after prolonged exposure to a near infrared laser, but takes roughly 15 minutes to reach optimal fluorescence [14]. Most importantly, because of fluorescein sodium's ability to analyze tumor cells in brain tissue, near-infrared confocal endomicroscopy allows microhistological information to be evaluated in real-time surgery, which can drastically improve how neurosurgeons proceed with malignant glioma surgery [14]. It is apparent that indocyanine green could potentially have a major impact on fluorescence-guided surgery, but more research must be conducted on the procedure before it can be integrated into standard glioma resection protocol.

Besides their use in a myriad of imaging techniques and drug therapy, nanoparticles can be utilized as exogenous contrast agents for enhancing tumor tissue during neurosurgery. Quantum dots, which are nanoparticles fabricated from semiconducting nanocrystals, can emphasize tumor regions and margins by fluorescence. Despite their ability to offer high contrast between tumor regions and healthy tissue, the controversial and unknown long-term ramifications of quantum dot use discourages neurosurgeons from utilizing it [8]. Other than quantum dots, polyamidoamine dendrimers, which are nanoparticles comprised of branched polymer chains that can be linked to fluorescent dyes, such as rhodamine B, or small organic molecules (methylene blue, Coomassie blue, or indocyanine green), to induce fluorescence in tumor tissue [8]. Polyamidoamine dendrimer use for fluorescence-guided surgery is appealing because this method can reduce the toxicity of some of the molecules, such as indocyanine green, that it is attached to. However, this technology has mainly been utilized for other usesimaging, targeted delivery, and therapeutic agents-and has yet to be studied extensively for its ability to label tumor tissue during brain surgery. Despite this, it will not be surprising if polyamidoamine dendrimer technology could prove to be successful in identifying brain tumors intraoperatively [8]. Lastly, iron oxide nanoparticles, which are superparamagnetic particles utilized in imaging tumors and cancer therapy, can also provide intraoperative fluorescence of tumors. CLIO-Cy5.5, an iron oxide nanoparticle developed for providing negative contrast for MRI, is capable of marking tumors intraoperatively and can also be utilized for preoperative MR imaging [8]. Because of this, CLIO-Cy5.5 could be utilized both preoperatively and intraoperatively. Also, iron oxide nanoparticles are capable of therapeutically targeting tumors while acting as biological markers [8]. It is evident that nanoparticle technology is capable of 
acting as an intraoperative fluorescent tumor marker; however, there has not been a significant amount of research on nanoparticles adopting this role and most studies surrounding medical nanoparticle technology have been stressed in other medical fields.

Activatable cell-penetrating peptides (ACPPs) are versatile targeting agents capable of aiding in a myriad of tasks preoperatively, intraoperatively, and postoperatively for tumor resection surgery. ACPPs consist of a region containing a polycationic cell-penetrating peptide linked to a polyanionic peptide, which neutralizes the positive charge, by a cleavable bond [15]. In order for ACPPs to enter their active state and translocate into tumor cells, the bond between the polyanionic peptide and the polycationic peptide, which is coupled to the cargo, must undergo proteolytic cleavage by matrix metalloproteinases (MMP). MMP2 and MMP9, which are proteases overexpressed in several types of cancer, are integral for tumorigenesis due to their ability to degrade extracellular matrices, induce angiogenesis, and promote growth factors [15]. The degree of MMP2 and MMP9 expression is contingent on the magnitude of tumor malignancy (the higher the grade of tumor, the higher expression of these proteins), and the enzymes have highly specific binding sites for substrates, thus making them robust targets for identifying cancer [15]. ACCPs can be conjugated to dendrimers (ACPPDs), which increases the degree of tumor fluorescence and contrast between the background and tumor, thus creating a superior delineation of tumor margins compared to ACPPs. ACPPDs can be conjugated with gadolinium-allowing tumor visualization by MRI before and after surgery. In a preclinical study on the efficacy of resection surgery guided by ACPPDs linked to Cy5, a fluorescent dye, and gadolinium, the authors found that this procedure on mice suffering from melanoma yielded a better prognosis (100\% higher eight month progression-free survival and 50\% higher overall survival) than mice subjected to white light surgery [15]. The drastic improvement in prognosis is due to a 10-fold increase in ACPPDs in subjects with complete resection compared to the group that underwent white light surgery and the procedure's 93\% specificity rate [15]. ACPPs can also aid in evaluating the presence of cancer in lymph nodes during surgery, which is vital to the progression of the procedure. Usually, in order to analyze the presence of cancer in lymph nodes, all lymph nodes in the anatomical vicinity of the primary tumor are surgically removed and pathologically examined in a process called sentinel lymph node (SLN) identification [16]. This strenuous, lengthy procedure is inefficient in providing feedback during surgery. In order to provide quick feedback regarding lymph node metastasis, ACPPs labeled with both Cy5 and Cy7 fluorophores-ratiometric ACPPs (RACPPs)-can rapidly identify cancer-containing lymph nodes intraoperatively. By linking Cy5-the fluorescent donor-to the polycationic peptide and attaching Cy7-the fluorescent acceptor-to the polyanionic domain, Förster resonance energy transfer interactions proceed due to the close proximity of the acceptor and donor when RACPP is in its deactivated, linked state. Separation of the donor from the acceptor, which occurs when MMP2 and MMP9 cleave RACPPs, induces dequenching fluorescence. Therefore, the fluorescence emitted from dequenching, which allows tumors to fluoresce significantly early during the procedure (one to two hours after injection), in conjunction with the accumulation of $\mathrm{Cy}-5$ labeled polycationic domains within tumor cells provides a more prolonged fluorescence with a higher tumor-background contrast compared to nonratiometric ACPPs, which take six and 24 hours for optimal use [16]. In a study conducted comparing the ability of non-ratiometric ACPPDs to RACPPs to detect metastases, RACPP analysis provided higher contrast between tumor tissue and healthy tissue compared to ACPPD contrast, despite the fact that significantly different intensities between tumor and healthy tissue was observed for both groups ( $p=.02$ compared to $p=.0007$ ) [16]. It is apparent that one can manipulate ACPPs in numerous ways by linking different types of cargo to ACPP domains to maximize the efficacy of resection surgeries. Overall, ACPPs could potentially have a major impact on resection surgery procedures for numerous types of cancer due to their dynamic nature and versatility.

\section{Conclusions}


Clearly, with all the fluorescent agents in our arsenal, radical changes must be implemented to improve glioma resections from standard white light surgery. By using white light surgery, surgeons make the subjective decision of delineating tumor margins. However, by using fluorescent-guided surgery, they will be better equipped to confidently and objectively discern between tumor tissue and healthy tissue. Ultimately, with the various methods of fluorescentguided surgery available, typical white light resection surgery will eventually become obsolete. Most of the fluorescent agents highlighted in this article have been utilized as biological markers; however, because the majority of agents used for fluorescent-guided surgery are merely in pre-clinical trials, their efficacy lacks significant research to implement them fully into contemporary surgical protocols.

\section{Additional Information \\ Disclosures}

Conflicts of interest: In compliance with the ICMJE uniform disclosure form, all authors declare the following: Payment/services info: All authors have declared that no financial support was received from any organization for the submitted work. Financial relationships: All authors have declared that they have no financial relationships at present or within the previous three years with any organizations that might have an interest in the submitted work. Other relationships: All authors have declared that there are no other relationships or activities that could appear to have influenced the submitted work.

\section{References}

1. Batchelor, T: Adjuvant Chemotherapy for Glioblastoma and Anaplastic Astrocytoma. UpToDate. 2014, Accessed: August 28, 2014: http://www.uptodate.com/contents/adjuvantchemotherapy-for-glioblastoma-and-anaplastic-astrocytoma\#H17.

2. Hefti M, Mehdorn M, Albert I, Dorner L: Fluorescence-Guided Surgery for Malignant Glioma: A Review on Aminolevulinic Acid Induced Protoporphyrin IX Photodynamic Diagnostic in Brain Tumors. Current Medical Imaging Reviews. 2010, 6:254-58. http://www.ingentaconnect.com/content/ben/cmir/2010/00000006/00000004/art00006.

3. Martirosyan NL, Georges J, Eschbacher JM, Cavalcanti DD, Elhadi AM, Abdelwahab MG, Scheck AC, Nakaji P, Spetzler RF, Preul MC: Potential application of a handheld confocal endomicroscope imaging system using a variety of fluorophores in experimental gliomas and normal brain. Neurosurg Focus. 2014, 36:E16. 10.3171/2013.11.FOCUS13486

4. Stummer W, Reulen HJ, Novotny A, Stepp H, Tonn JC: Fluorescence-guided resections of malignant gliomas--an overview. Acta Neurochir Suppl. 2003, 88:9-12.

5. Zehri AH, Ramey W, Georges JF, Mooney MA, Martirosyan NL, Preul MC, Nakaji P: Neurosurgical confocal endomicroscopy: A review of contrast agents, confocal systems, and future imaging modalities. Surg Neurol Int. 2014, 5:60. 10.4103/2152-7806.131638

6. Widhalm G, Kiesel B, Woehrer A, Traub-Weidinger T, Preusser M, Marosi C, Prayer D, Hainfellner JA, Knosp E, Wolfsberger S: 5-Aminolevulinic acid induced fluorescence is a powerful intraoperative marker for precise histopathological grading of gliomas with nonsignificant contrast-enhancement. PLoS ONE. 2013, 8:E76988. 10.1371/journal.pone.0076988

7. Della Puppa A, Ciccarino P, Lombardi G, Rolma G, Cecchin D, Rossetto M: 5-Aminolevulinic acid fluorescence in high grade glioma surgery: surgical outcome, intraoperative findings, and fluorescence patterns. Biomed Res Int. 2014, 2014:232561. 10.1155/2014/232561

8. Pogue BW, Gibbs-Strauss S, Valdés PA, Samkoe K, Roberts DW, Paulsen KD: Review of neurosurgical fluorescence imaging methodologies. IEEE J Sel Top Quantum Electron. 2010, 16:493-505.

9. Friesen SA, Hjortland GO, Madsen SJ, Hirschberg H, Engebraten O, Nesland JM, Peng Q: 5Aminolevulinic acid-based photodynamic detection and therapy of brain tumors (review). Int J Oncol. 2002, 21:577-82.

10. Piquer J, Llácer JL, Rovira V, Riesgo P, Rodriguez R, Cremades A: Fluorescence-guided surgery and biopsy in gliomas with an exoscope system. Biomed Res Int. 2014, 2014:207974.

$10.1155 / 2014 / 207974$ 
11. Hebeda KM, Saarnak AE, Olivo M, Sterenborg HJ, Wolbers JG: 5-Aminolevulinic acid induced endogenous porphyrin fluorescence in 9L and C6 brain tumours and in the normal rat brain. Acta Neurochir (Wien). 1998, 140:503-12.

12. Lin WC, Toms SA, Johnson M, Jansen ED, Mahadevan-Jansen A: In vivo brain tumor demarcation using optical spectroscopy. Photochem Photobiol. 2001, 73:396-402.

13. Acerbi F, Broggi M, Eoli M, Anghileri E, Cavallo C, Boffano C, Cordella R, Cuppini L, Pollo B, Schiariti M, Visintini S, Orsi C, La Corte E, Broggi G, Ferroli P: Is fluorescein-guided technique able to help in resection of high-grade gliomas?. Neurosurg Focus. 2014, 36:E5. 10.3171/2013.11.FOCUS13487

14. Martirosyan NL, Cavalcanti DD, Eschbacher JM, Delaney PM, Scheck AC, Abdelwahab MG, Nakaji P, Spetzler RF, Preul MC: Use of in vivo near-infrared laser confocal endomicroscopy with indocyanine green to detect the boundary of infiltrative tumor. J Neurosurg. 2011, 115:1131-8. 10.3171/2011.8.JNS11559

15. Nguyen QT, Olson ES, Aguilera TA, Jiang T, Scadeng M, Ellies LG, Tsien RY: Surgery with molecular fluorescence imaging using activatable cell-penetrating peptides decreases residual cancer and improves survival. Proc Natl Acad Sci USA. 2010, 107:4317-22. 10.1073/pnas.0910261107

16. Savariar EN, Felsen CN, Nashi N, Jiang T, Ellies LG, Steinbach P, Tsien RY, Nguyen QT: Realtime in vivo molecular detection of primary tumors and metastases with ratiometric activatable cell-penetrating peptides. Cancer Res. 2013, 73:855-64. 10.1158/0008-5472.CAN12-2969 